\title{
钯/降冰片烯/手性氨基酸协同催化立体选择性构建全碳桥环分子骨架
}

\author{
叶金祥周强辉* \\ (武汉大学化学与分子科学学院 武汉 430072)
}

\section{Pd/NBE/Chiral Amino Acid Cooperative Catalysis for Enantioselective Construction of All-Carbon Brideged Ring Systems}

\author{
Ye, Jinxiang Zhou, Qianghui* \\ (College of Chemistry and Molecular Sciences, Wuhan University, Wuhan 430072)
}

钯/降冰片烯(NBE)协同催化是一种制备多取代芳 烃的策略, 由 Catellani 小组 ${ }^{[1]}$ 在 1997 年首次报道, 因此 被称为 Catellani 反应. Catellani 反应将经典的交叉偶联 反应和 $\mathrm{C}-\mathrm{H}$ 键活化有效连接起来, 能对卤代芳烃(主要 是碘代芳烃)邻位 $\mathrm{C}-\mathrm{H}$ 键和原位 $\mathrm{C}-\mathrm{X}$ 键进行连续的官 能团化, 一次构建多根化学键, 合成一系列其他方法难 以合成的多取代芳烃和芳并环结构单元. 经过 20 多年 的发展, 尤其是近年来通过开发降冰片烯衍生物作为新 型的助催化剂, Catellani 反应的应用得到了极大拓展, 底物适用范围方面也突破了卤代芳烃体系.

考虑到 Catellani 反应在制备多取代芳烃方面的优 势, 人们期待发展催化不对称 Catellani 反应, 用于制备 手性的多取代芳烃. 然而, 由于钯/降冰片烯协同催化过 程复杂, 催化不对称 Catellani 反应的研究一直未有突破. 直到 2016 年, 这个方向才取得了进步 ${ }^{[2]}$. 目前报道的催 化不对称 Catellani 反应主要有两种立体选择性控制模 式: 手性膦配体参与控制不对称的原位终止反应和手性 降冰片烯衍生物 NBE*作为助催化剂控制. 顾振华课题 组 ${ }^{[3]}$ 率先发展了富电子大位阻手性膦配体调控 Catellani 反应的不对称终止策略(图 1a), 成功应用于 $(+)$ Rhazinal 家族生物碱的不对称全合成研究和构建联芳基 轴手性. 而手性 $\mathrm{NBE}^{*}$ 立体调控模式的核心在于构建手 性五元环钯络合物中间体(ANP*), 实现立体选择性的 氧化加成和还原消除, 从而在产物中构建手性元素(图 1a). 2018 年, 余金权课题组报道了钯/手性降冰片烯协 同催化的芳烃间位 $\mathrm{C}-\mathrm{H}$ 键芳基化和烷基化, 通过去对 称化或动力学拆分过程实现了高立体选择性远程构筑 手性中心 ${ }^{[4]}$. 同年, 董广涁课题组 ${ }^{[5]}$ 报道了钯/手性降冰
片烯协同催化对环氧化合物的动力学拆分, 但立体选择 性不高. 随后，周强辉课题组 ${ }^{[6]}$ 将钯/手性降冰片烯协同 催化策略应用于构建联芳基轴手性和对叔茮醇的动力 学拆分 ${ }^{[7]}$, 并通过手性转移策略实现了手性芴醇和 $\mathrm{C}$ $\mathrm{N}$ 轴手性的高效构建 ${ }^{[8]} .2021$ 年, 宋秋玲课题组 ${ }^{[9]}$ 用类似 的策略实现了联芳基轴手性膦氧化合物的合成.

尽管上述两种立体选择性控制模式的催化不对称 Catellani 反应已经取得了不错的进展, 发展更加高效、 底物适用范围广的新型立体化学控制模式依然是该领 域迫切需要解决的问题. 近期, 中国科学技术大学化学 系龚流柱课题组 ${ }^{[10]}$ 发展了一种 $\mathrm{Pd} / \mathrm{NBE} /$ 手性氨基酸协同 催化的新型不对称 Catellani 环化反应, 使用手性氨基酸 催化剂作为手性源(图 1a), 高效构建了一系列含全碳桥 环骨架的手性化合物, 快速增加分子的复杂度. 他们设 想的反应机理如图 $1 b$ 所示, 碘代底物首先发生邻位 C$\mathrm{H}$ 键活化反应, 形成五元环钯中间体 I, 然后与 4-(溴甲 基)环丁酮的 $\mathrm{C}-\mathrm{Br}$ 键发生氧化加成、还原消除得到邻 位烷基化中间体 II. 中间体 II 的酮羰基和手性氨基酸催 化剂原位生成烯胺中间体，同时，手性氨基酸上羧基与 钯中心配位形成关键手性中间体 III, 继而发生不对称 $\alpha$-芳基化终止反应得到目标产物. 值得一提的是，周强 辉课题组 ${ }^{[11]}$ 在 2019 年报道了通过 $\mathrm{Pd} / \mathrm{NBE} /$ 手性胺协同 催化，促进碘代芳烃和 4-苯酰氧胺基环己酮之间的不对 称 Catellani 环化反应来构建氮杂桥环化合物, 但对映选 择性不高.

龚流柱课题组选取 $\alpha$-碘芸与 4-(溴甲基)环己酮之间 的反应作为模板反应进行研究. 通过一系列反应条件的 考察, 确认 $(S)-2$-吲哚啉羧酸为最优的手性氨基酸催化

* Corresponding author. E-mail: qhzhou@whu.edu.cn. Published online December 9, 2021. 
(a) Enantio-determing step of asymmetric Catellani-type reactions

(i) Chiral ligand strategy

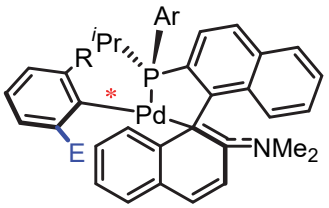

$\mathrm{Gu}, 2016,2018$ (ii) Chiral $\mathrm{NBE}^{*}$ strategy

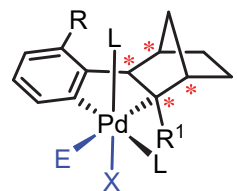

Yu, Dong, Zhou, Song (since 2018) (iii) Chiral amine strategy

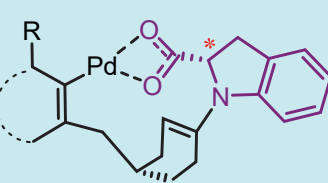

Gong, 2021

(b) Assembly of all-carbon bridged ring via $\mathrm{Pd} / \mathrm{NBE} /$ chiral amine cooperative catalysis<smiles>[R]C1CCCC=C1I</smiles>

O. A./

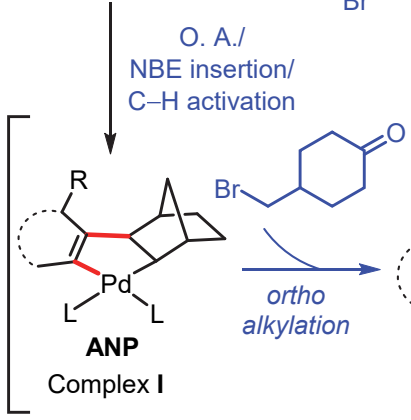

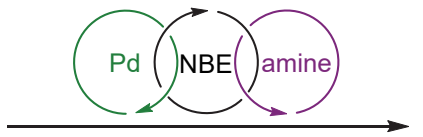<smiles>[R]C1CCCC2=C1[C@@H]1CC(=O)CCC[C@H]1C2</smiles>

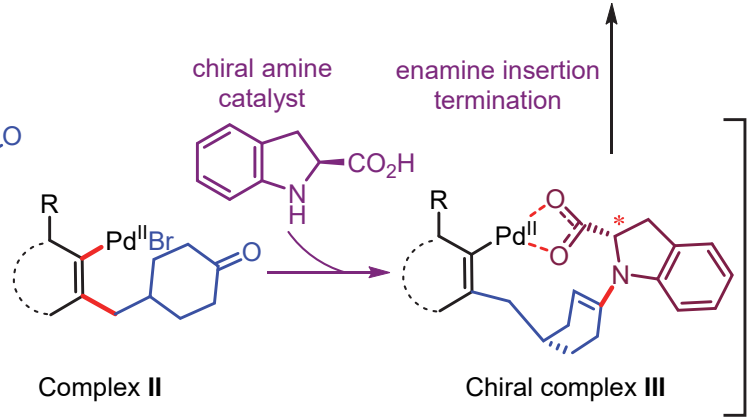

图 1 不对称 Catellani 反应

Figure 1 Asymmetric Catellani-type reactions

剂，与钯催化剂和降冰片烯衍生物 5-氰基降冰片烯 $\left(\mathbf{N}^{\mathbf{1}}\right)$ 或 2-甲酰胺降冰片烯 $\left(\mathbf{N}^{2}\right)$ 协同作用, 取得最好的反应效 果 $(67 \%, 97 \% e e)$. 该类反应具有较广的底物适用范围, 芳烃碘化物、4-碘代喹啉酮和烯基三氟甲磺酸酯三类底 物都能在类似的反应条件下与 4-(溴甲基)环已酮发生反 应，以中等到良好的收率和优秀的立体选择性(up to 97\% ee)得到相应含全碳桥环骨架的目标产物(Scheme 1). 最后, 龚流柱课题组通过一系列对照实验和密度泛 函理论(DFT)计算对反应的机理进行了深入研究，证明 手性氨基酸催化剂 $(S)$-2-吲哚啉羧酸在提高反应效率以

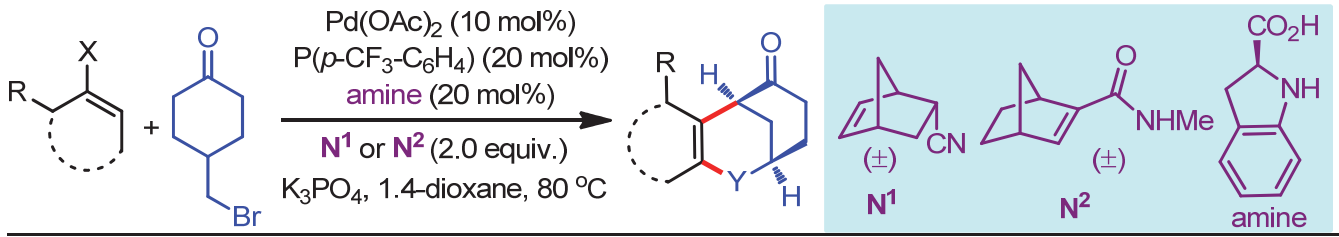<smiles>C[C@H]1CCC(=O)c2c(ccc3ccccc23)C1</smiles>

$69 \%$ yield, $95 \%$ ee<smiles>O=C1CC[C@H]2Cc3ccc4c(Br)cccc4c3[C@H]1C2</smiles>

$53 \%$ yield, $96 \%$ ee<smiles>O=C1CC[C@H]2CC3=C(c4ccccc4CC3)[C@H]12</smiles><smiles>CCC1=C2[CH]CCC(=O)[C@@H](C2)c2ccc(Br)c(F)c2CC1</smiles>

$34 \%$ yield, $90 \%$ ee<smiles>O=C1CC[C@H]2Cc3cc4c5c(cccc5c3[C@H]1C2)CC4</smiles>

$75 \%$ yield, $97 \%$ ee

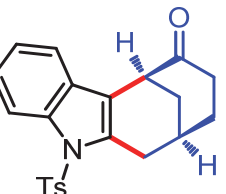

$48 \%$ yield, $91 \%$ ee<smiles>Cn1c(=O)c2c(c3ccccc31)[C@H]1C[C@H](CCC1=O)CC2</smiles>

$95 \%$ yield, $97 \%$ ee

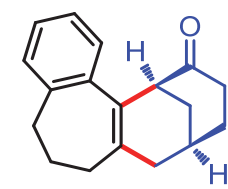

$30 \%$ yield, $91 \%$ ee<smiles>O=C1CC[C@@H]2Cc3ccc4c(sc5ccccc54)c3[C@H]1C2</smiles>

$51 \%$ yield, $95 \%$ ee<smiles>O=C1CC[C@H]2Cc3c(c(=O)n(Cc4ccccc4)c4cc5c(cc34)OCO5)C[C@H]1C2</smiles>

$42 \%$ yield, $84 \%$ ee

图式 1 代表性全碳桥环骨架产物

Scheme 1 Representative products with all-carbon brideged ring system 
及立体选择性控制方面均起着关键作用. (S)-2-吲哚啉 羧酸作为双官能有机催化剂, 一方面通过氨基将嶡基烯 胺化提高终止反应的效率, 另一方面通过羧基与 $\mathrm{Ar}-$ $\mathrm{Pd}^{\mathrm{II}}$ 中心有效配位确保了终止反应的高立体选择性.

综上所述, 龚流柱课题组发展了钯/ $\mathrm{NBE} /$ 手性氨基 酸协同催化的一种新型不对称 Catellani 环化反应, 高对 映选择性地构建结构复杂的全碳桥环骨架化合物。该策 略将手性有机催化剂引入 Pd/NBE 协同催化反应的终止 阶段, 在加速终止反应的同时将手性元素高效地引入产 物, 丰富了不对称 Catellani 反应的立体化学控制模式.

\section{References}

[1] Catellani, M.; Frignani, F.; Rangoni, A. Angew. Chem., Int. Ed.
$1997,36,119$.

[2] Zhao, K.; Xu, S.; Pan, C.; Sui, X.; Gu. Z. Org. Lett. 2016, 18, 3782.

[3] Ding, L.; Sui, X.; Gu, Z. ACS Catal. 2018, 8, 5630.

[4] Shi, H.; Herron, A. N.; Shao, Y.; Shao, Q.; Yu, J.-Q. Nature 2018, $558,581$.

[5] Li, R.; Liu, F.; Dong, G. Org. Chem. Front. 2018, 5, 3108.

[6] Liu, Z.-S.; Hua, Y.; Gao, Q.; Ma, Y.; Tang, H.; Shang, Y.; Cheng, H.-G.; Zhou, Q. Nat. Catal. 2020, 3, 727.

[7] Hua, Y.; Liu, Z.-S.; Xie, P.-P.; Ding, B.; Cheng, H.-G.; Hong, X.; Zhou, Q. Angew. Chem., Int. Ed. 2021, 60, 12824.

[8] Liu, Z.-S.; Xie, P.-P.; Hua, Y.; Wu, C.; Ma, Y.; Chen, J.; Cheng, H.-G.; Hong, X.; Zhou, Q. Chem 2021, 7, 1917.

[9] Feng, Q.; Ma, X.; Bao, W.; Li, S.; Lan. Y.; Song, Q. CCS Chem. 2021, 3, 377.

[10] Chen, X.; Zhu, L.; Chen, D.; Gong, L. Angew. Chem., Int. Ed. 2021, $60,24844$.

[11] Gao, Q.; Liu, Z.-S.; Hua, Y.; Li, L.; Cheng, H.-G.; Cong, H.; Zhou, Q. Chem. Comm. 2019, 55, 8816.

(Lu, Y.) 